Check for updates

Cite this: Mater. Adv., 2022, 3, 2117

Received 26th October 2021 Accepted 9th January 2022

DOI: $10.1039 / \mathrm{d} 1 \mathrm{ma00992c}$

rsc.li/materials-advances

\title{
The impact of the isomerism of peptide mimetics on their assembly and properties: quick and onsite gas phase detection of acids and alcohols $\dagger$
}

\author{
Deepta Chattapadhyay, Sujay Kumar Nandi and Debasish Haldar (DD *
}

\begin{abstract}
The effect of isomerism on the structure, self-assembly and properties of two peptide mimetics has been investigated. These peptide mimetics contain $m$-aminobenzoic acid, $p$-aminobenzoic acid and $N, N^{\prime}$-dicyclohexylurea. From $\mathrm{X}$-ray crystallography, it is observed that the $p$-isomer adopted a kink-like conformation stabilized by $\mathrm{C}-\mathrm{H} \cdots \pi$ interactions and formed a supramolecular anti-parallel duplex and layer by layer sheet-like structure in higher order assembly. However, only the $m$-isomer formed sonication responsive gels in different hydrocarbons such as hexane-EtOAc (19:1), diesel, kerosene, body oil and coconut oil. From rheology experiments, the gel was found to have physical cross-links and is elastic in nature. The FE-SEM images depicted that the $m$-isomer exhibited nanofiber network morphology, whereas the $p$-isomer exhibited polydisperse microsphere morphology. The gel was found to be highly sensitive to alcohol vapours, although the kinetics become much slower from methanol to ethanol to isopropanol. The gel is also very responsive to acid vapours like $\mathrm{HCl}$.
\end{abstract}

\section{Introduction}

Pollution is a by-product of industrialization and traffic, and the sensing and control of pollutants are important for preserving the nature and protecting the human life. ${ }^{1}$ Volatile organic compounds (VOCs) from the industrial and car exhaust constitute a major fraction of the polluted air. ${ }^{2}$ Therefore naked eye monitoring of the VOC concentration in air is highly important to ensure healthy living conditions. In general VOCs can be both aliphatic as well as aromatic in nature. For example, acetone, a highly volatile aliphatic solvent, is widely used in industries and research laboratories. But a high amount of acetone in air can cause neurological damages, affect kidneys, create diabetes-like symptoms, for example hyperglycemia and glycosuria, and cause cardiovascular damage. ${ }^{3}$ THF is another commonly used aliphatic organic solvent. It is highly carcinogenic and causes severe irritation in the eyes, lungs, liver and kidneys. ${ }^{4}$ Chlorinated aliphatic solvents like chloroform and dichloromethane directly affect the central nervous system. ${ }^{5}$ Aromatic organic solvents like benzene, toluene, xylenes, etc. are extremely carcinogenic in nature. ${ }^{6} \mathrm{HCl}$ vapour is produced

Department of Chemical Sciences, Indian Institute of Science Education and Research Kolkata, Mohanpur-741246, West Bengal, India.

E-mail: deba_h76@yahoo.com,deba_h76@iiserkol.ac.in

$\dagger$ Electronic supplementary information (ESI) available: Peptide synthesis, NMR spectroscopy, Fig. ESI S1-S16, and peptide single crystals. CCDC 2006754. For ESI and crystallographic data in CIF or other electronic format see DOI: 10.1039/ d1ma00992c by the iron and steel industry, the chemical industry and the plastic recycling industry and with long-time exposure, $\mathrm{HCl}$ vapours may cause irritation in the respiratory system and eyes and lead to damage of mucosa. ${ }^{7}$ So, the detection of VOCs in the gas phase as well as acid vapour is extremely necessary for sustainability.

Stimuli responsive materials have attracted huge attention due to their selective sensing ability. The inherent properties (volume, color, or viscosity) of these materials change by sensing an analyte. ${ }^{8}$ Among different stimuli responsive materials, the ones whose fluorescence output can be tuned by external stimuli like $\mathrm{pH}$, ions, light, temperature, redox potential, and vapours are highly promising. ${ }^{9}$ These luminescent materials are already being used in sensing devices, memory and display devices, for the fabrication of detectors and in thermal imaging. ${ }^{10-12}$ In this context, the development of a VOC responsive self-assembled smart material will be very useful. Self-assembly is generally driven by various secondary interactions such as hydrogen bonding, $\pi-\pi$ stacking, $\mathrm{C}-\mathrm{H} \cdots \pi$ interactions, dipole-dipole interactions, and van der Waals interactions. Earlier, different groups have reported VOC sensors based on cavitands, ${ }^{13,14}$ metal-organic frameworks, ${ }^{15,16}$ luminescent materials, ${ }^{17,18}$ and organic vapochromic materials. ${ }^{19,20}$

Previously we have reported an on-line ammonia sensor and invisible security ink using a fluorescent zwitterionic spirocyclic Meisenheimer complex. ${ }^{21}$ We have also shown that a selfhealing hydrogel from a dipeptide can act as a $\mathrm{HCl}$ sensor. $^{22}$ 
Recently, we have reported a robust tripeptide for in-field selective naked eye ultratrace detection of 2,4,6-trinitrophenol. ${ }^{23}$ Herein, we have reported the effect of isomerism on the structure, self-assembly and properties of two peptide mimetics. From X-ray crystallography, it is observed that the $p$-isomer adopted a kink-like conformation stabilized by $\mathrm{C}-\mathrm{H} \cdots \pi$ interactions and formed a supramolecular anti-parallel duplex and layer by layer sheet-like structure in higher order assembly. Interestingly, the $m$-isomer formed sonication induced organogels in different hydrocarbons such as hexaneEtOAc (19:1), diesel, kerosene, body oil and coconut oil. From rheology experiments, the gel is found to have physical cross-links and to be elastic in nature. FE-SEM images depicted that the $m$-isomer exhibited nanofiber network morphology, whereas the $p$-isomer exhibited polydisperse microsphere morphology. The gel was found to be highly sensitive to alcohols. However, the kinetics become much slower from methanol to ethanol to isopropanol. The gel is also very responsive to acid vapors like $\mathrm{HCl}$.

\section{Results and discussion}

We have designed and synthesized a series of compounds with $m$-aminobenzoic acid, $p$-nitrobenzoic acid and $N, N^{\prime}$ dicyclohexylurea by a conventional solution phase methodology (ESI, $\dagger$ Fig. S1). ${ }^{24,25}$ Since $N, N^{\prime}$-dicyclohexylurea contains several intermolecular hydrogen bonding sites, we thought the $\pi-\pi$ interactions on the aromatic ring in addition to the H-bonding could significantly modulate the self-assembly behaviour of the compounds. Peptide mimetics $\mathbf{1}$ and $\mathbf{2}$ are isomers (Scheme 1). We wish to study the effect of isomerism on the structure, selfassembly and properties of the peptide mimetics.

The final compounds have been purified by column chromatography and characterized by ${ }^{1} \mathrm{H}-\mathrm{NMR},{ }^{13} \mathrm{C}-\mathrm{NMR}$, FT-IR and mass spectrometry analysis. Peptide mimetic 2 was also characterized by single crystal X-ray diffraction analysis. We have failed to develop crystals of peptide 1 suitable for X-ray crystallography. Peptide mimetic 2 crystallizes with one molecule in the asymmetric unit from methanol-water solution by slow evaporation (Fig. 1a). The compound adopted a kink like structure and is stabilized by $\mathrm{C}-\mathrm{H} \cdots \pi$ interactions (Fig. 1a). The $\mathrm{C}-\mathrm{H}$ to centroid distance is $3.9 \AA$. There is no intramolecular hydrogen bond. Moreover, the peptide mimetic 2 self-assembles through reciprocal intermolecular hydrogen

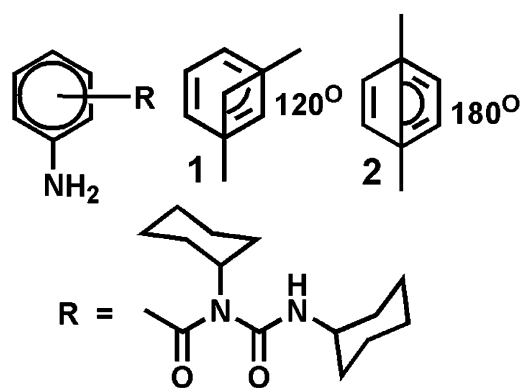

Scheme 1 The schematic representation of isomeric peptide mimetics 1 and 2 .

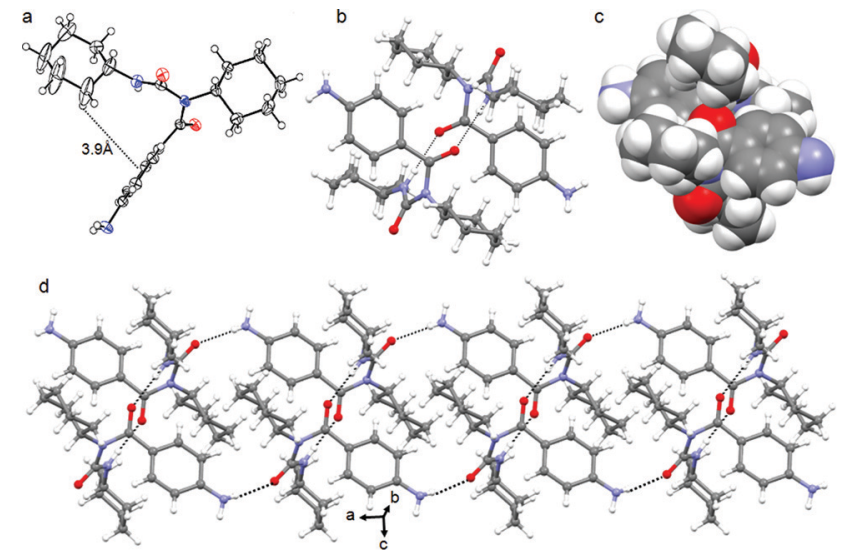

Fig. 1 (a) The ORTEP diagram of peptide mimetic 2. Probability $50 \%$. The $\mathrm{C}-\mathrm{H} \cdots \pi$ interaction is shown as black dotted lines. (b) The intermolecular hydrogen bonded anti-parallel duplex of peptide mimetic 2. Hydrogen bonds are shown as black dotted lines. (c) The space fill model of the duplex along the crystallographic a axis showing intermolecular $\mathrm{C}-\mathrm{H} \cdots \pi$ interactions. (d) 1D array of peptide mimetic 2 through intermolecular hydrogen bonding interactions along the crystallographic a direction. Hydrogen bonds are shown as black dotted lines.

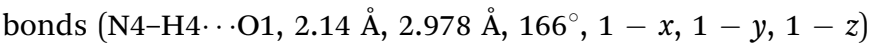
to form an anti-parallel duplex structure (Fig. 1b). The duplex is also stabilized by intermolecular $\mathrm{C}-\mathrm{H} \cdots \pi$ interactions (Fig. 1c). The packing diagram of peptide mimetic 2 shows that the dimers further self-assemble to form a supramolecular 1D structure through intermolecular hydrogen bonds (N5HA ‥ 2 2, $2.32 \AA, 3.123 \AA, 156^{\circ}, 1-x, 1-y, 2-z$ and

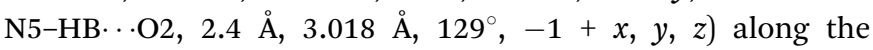
crystallographic $a$ axis (Fig. 1d).

In higher order packing, peptide mimetic 2 forms a layer by layer sheet-like structure (Fig. 2) along crystallographic $a$ and $b$ directions. Though there is no $\pi-\pi$ stacking interaction, the aromatic rings formed one layer which is sandwiched between two hydrophobic cyclohexane layers (Fig. 2).

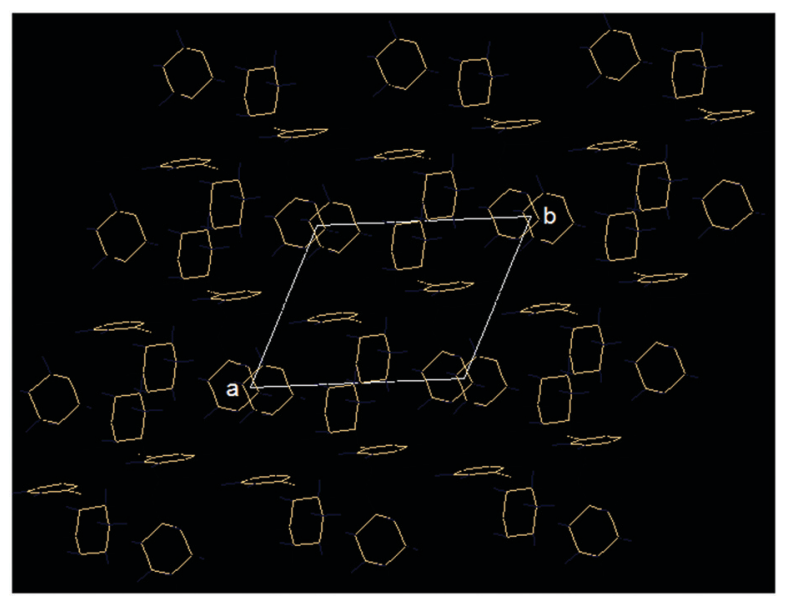

Fig. 2 (a) The higher order packing diagram of peptide mimetic 2 showing the layered structure along crystallographic $a$ and $b$ directions. The aromatic rings are sandwiched between two hydrophobic cyclohexane layers. 
Initially, the gelation abilities of the peptides were investigated in various organic solvents by dissolving $10 \mathrm{mg}$ of compound in $1 \mathrm{~mL}$ of solvent followed by the heating-cooling technique. But the compounds did not form gels (ESI, † Fig. S2). But on sonication for 20 minutes, we observed that peptide mimetic 1 formed organogels in hexane. However, on addition of $5 \%(\mathrm{v} / \mathrm{v})$ ethyl acetate, the sonication-induced gelation occurred instantly (Fig. 3a). The gel formation was confirmed by an inverted vial test. Peptide mimetic $\mathbf{1}$ also formed gels in different oils such as diesel, kerosene, body oil and coconut oil after sonicating for 1-2 minutes (Fig. 3). The gels are opaque in nature. The minimum gelation concentration (MGC) for peptide mimetic 1 was found to be $5 \mathrm{mg} \mathrm{mL}{ }^{-1}$. The peptide mimetic-based sonication responsive gel showed high stability for a couple of weeks. Similar conditions were tried for the $p$-isomer 2, but it failed to show any gelation behaviour in different organic solvents even at high concentrations.

Rheology experiments were performed to measure the mechanical strength of the organogel. Rheology data were taken as a function of angular frequency and oscillatory strain (Fig. 4). In rheology, there are two main parameters, the elastic response which is measured using storage modulus $G^{\prime}$ and the viscous response which is measured using loss modulus $G^{\prime \prime}$. In the frequency sweep experiment, the storage modulus $G^{\prime}$ is one order of magnitude higher than the loss modulus $G^{\prime \prime}$ over the entire angular frequency range, which confirms the formation of the elastic gel by physical cross-linking through weak interactions (Fig. 4a). In the amplitude sweep experiment, the loss modulus $G^{\prime \prime}$ becomes greater than storage modulus $G^{\prime}$ at $1.1 \%$ oscillation strain, which indicates that the gel breaks after this strain (Fig. 4b).

Peptide mimetic 1 gels exhibit amazing self-healing ${ }^{26}$ properties. When we placed two pieces of a gel together, one in body oil and the other in kerosene oil, we observed, to our surprise, that the two oils slowly got mixed together. Within 12 hours, two gel bars formed a single bar and the oils were completely mixed with each other indicating the self-healing nature of the gel (ESI, $\dagger$ Fig. S3). The self-healing may be due to the dynamic equilibrium between the generation of the new fibers and the disconnection of the old fibers and that governs the reformation of the gel. But the organogel is not thixotropic.
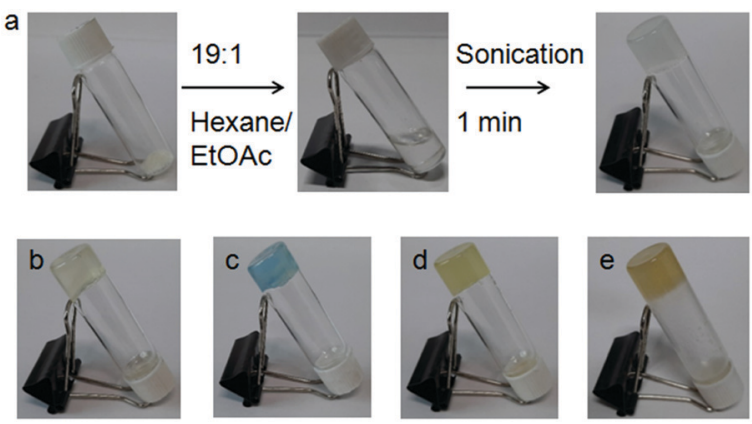

Fig. 3 (a) Sonication-induced gels of peptide mimetic 1 in hexane/ethyl acetate mixture. The sonication-induced gels of peptide mimetic $\mathbf{1}$ in (b) diesel, (c) kerosene oil, (d) body oil and (e) coconut oil.
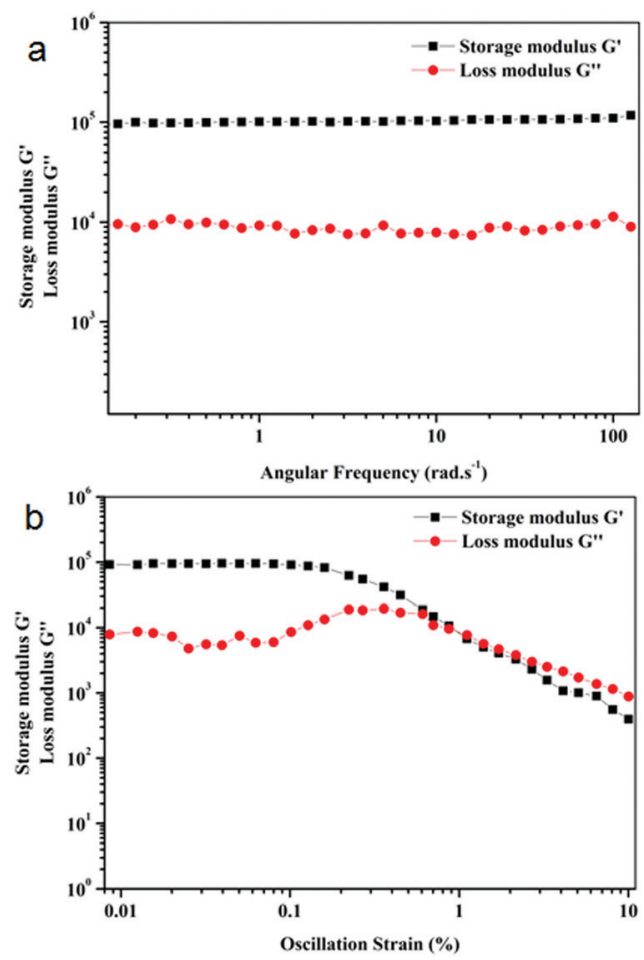

Fig. 4 Rheology of organogels of peptide mimetic 1 in $19: 1$ hexane/ethyl acetate, (a) frequency sweep experiment and (b) oscillation strain.

The morphologies of the peptide mimetic 1 gels were examined using a polarized optical microscope (POM). The xerogels of the organogel formed in the hexane/EtOAc mixture show a micro-fibre like morphology under a polarized optical microscope (ESI, $\dagger$ Fig. S4a). The morphology of peptide mimetic 2 was also investigated. For that, a drop of the peptide mimetic 2 solution in hexane was placed on a microscopic glass slide and allowed to dry at room temperature for one day. It was observed that peptide mimetic 2 forms microsphere like morphology (ESI, $\dagger$ Fig. S4b). To better understand the morphologies of the gels, field emission scanning electron microscopy (FE-SEM) is a wonderful technique. From FE-SEM, xerogels of peptide mimetic 1 obtained from hexane/EtOAc show the branch of nanofibers of length up to few micrometers (Fig. $5 \mathrm{a}$ and b). The average diameter of the fibers is $25 \mathrm{~nm}$. The nanofibers are interlinked to produce a $3 \mathrm{D}$ network that can entrap solvents (Fig. 5b). From FE-SEM images, it was observed that peptide mimetic 2 forms polydisperse microsphere like morphology (Fig. 5c and d). The average diameter of the microspheres is $300 \mathrm{~nm}$.

Only peptide mimetic $\mathbf{1}$ forms an organogel, which indicates that the $\mathrm{H}$-bonding through the aromatic amine group might play a crucial role in gelation. This fact is further supported by the observation that on addition of a polar protic solvent, the gel breaks. FT-IR spectroscopy is a very useful technique to probe this, since H-bonded amine gives a different signal from that of a free amine. The FT-IR spectra of the as-synthesized peptide mimetic 1 in the $\mathrm{KBr}$ pellet show three peaks due to $\mathrm{NH}$ stretching. The two peaks at $3298 \mathrm{~cm}^{-1}$ and $3348 \mathrm{~cm}^{-1}$ 


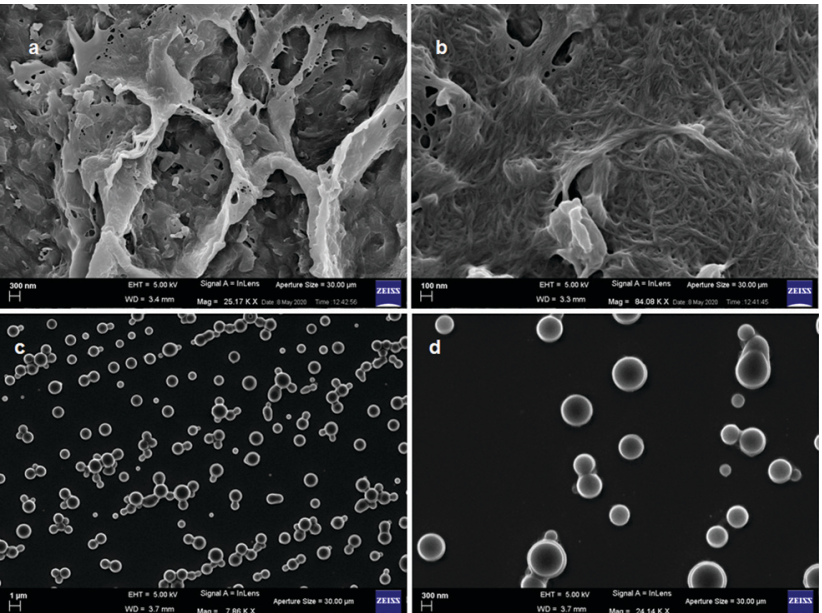

Fig. 5 ( $a$ and b) FE-SEM images of the xerogels of peptide 1 from hexane/ EtOAc showing nanofiber network morphology. (c and d) FE-SEM images of peptide $\mathbf{2}$ showing polydisperse microsphere morphology.

correspond to the aromatic amine stretching whereas the peak at $3438 \mathrm{~cm}^{-1}$ corresponds to the non-hydrogen bonded amide NH stretching (Fig. 6). The IR spectra of xerogels show a broad signal with a peak at around $3341 \mathrm{~cm}^{-1}$ indicating the presence of hydrogen bonded NH. This supports the role of $\mathrm{H}$-bonding in sonication-induced gelation of peptide mimetic 1 . The two separate carbonyl peaks at $1682 \mathrm{~cm}^{-1}$ and $1634 \mathrm{~cm}^{-1}$ for peptide mimetic 1 also get broadened and appear as one peak at around $1630 \mathrm{~cm}^{-1}$ for the xerogel (Fig. 6). The peptide mimetic 2 shows $\mathrm{NH}$ stretching at $3369 \mathrm{~cm}^{-1}$ and $3312 \mathrm{~cm}^{-1}$ corresponding to the hydrogen bonded $\mathrm{NH}$ and amide I and amide II at $1680 \mathrm{~cm}^{-1}$ and $1540 \mathrm{~cm}^{-1}$.

Moreover, the peptide mimetic $\mathbf{1}$ organogel can be used as a sensor for volatile acids. This is very interesting for pollutant detection, the automotive industry and the chemical industry. $\mathrm{HCl}$ vapor was charged to the organogel of peptide mimetic 1; on contact with $\mathrm{HCl}$ the gel transforms to a sol (Fig. 7). In the

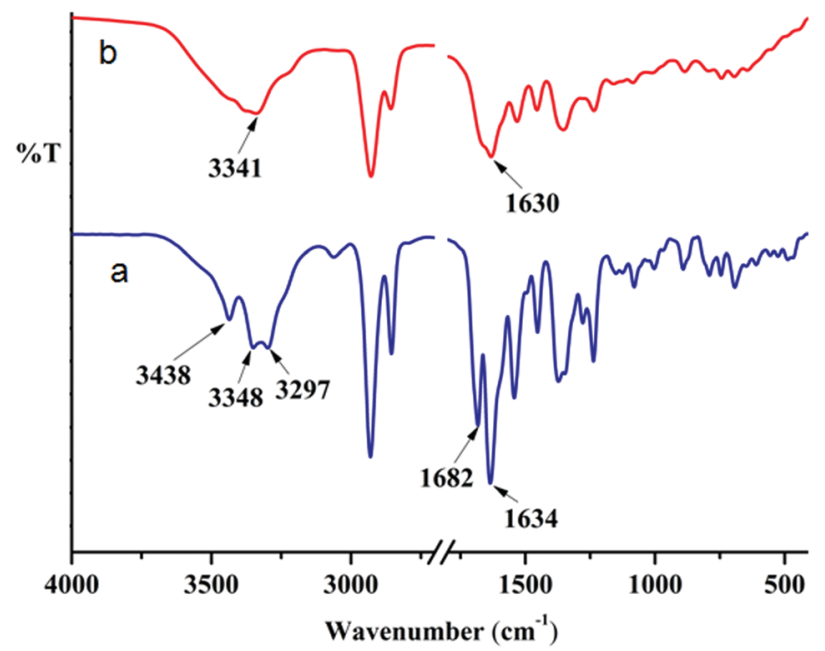

Fig. 6 FT-IR spectra of (a) as-synthesized peptide mimetic 1 and (b) peptide mimetic 1 xerogel.
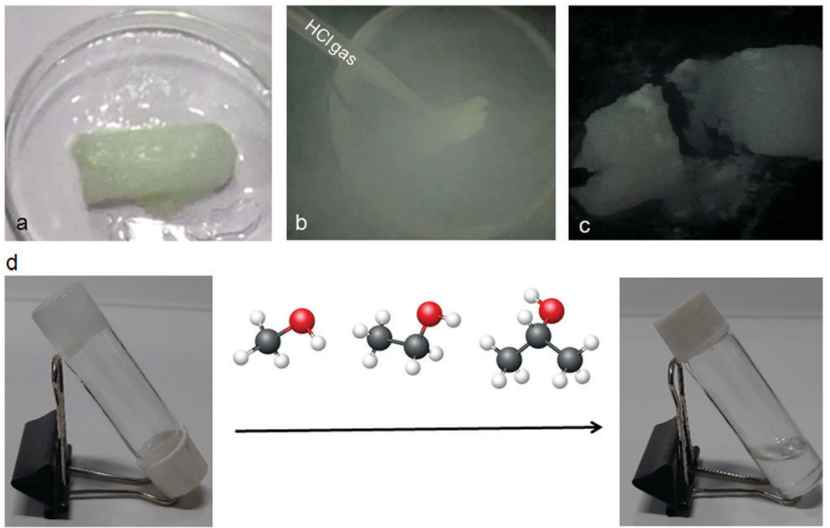

Fig. 7 (a-c) Sensitivity of the peptide mimetic 1 organogel towards $\mathrm{HCl}$ gas. (d) Sensitivity of the peptide mimetic 1 organogel towards alcohols.

presence of $\mathrm{HCl}$, the amine functional group of the gelator gets protonated and the gel transforms to sol. The gel also breaks on addition of acids such as $\mathrm{H}_{2} \mathrm{SO}_{4}$ and $\mathrm{HNO}_{3}$ (ESI, $\dagger$ Fig. S5). The gel is sensitive to acetic acid but not to formic acid, ammonia, methyl amine and ethyl amine (ESI, $\uparrow$ Fig. S5). The process of acid vapour sensing using the peptide mimetic $\mathbf{1}$ organogel can be upgraded to an on-line process for safety related application in industries which use or produce large amounts of concentrated acids. ${ }^{21}$

It is interesting to note that the organogel of the peptide mimetic 1 was found to be highly sensitive towards alcohols. Even, the gel broke immediately after addition of as little as $10 \mu \mathrm{L}$ of $\mathrm{MeOH}$. However, it was not specific to methanol only; ethanol and isopropanol could also break the gel (Fig. 7d). The kinetics become much slower as we go from methanol to ethanol to isopropanol. However, the gel remains intact when other organic solvents such as DCM and acetone are added.

\section{Experimental}

\section{General}

All chemicals and reagents were purchased from Sigma chemicals. HOBt (1-hydroxybenzotriazole) and DCC (dicyclohexylcarbodiimide) were purchased from SRL.

\section{Synthesis}

All the compounds were synthesized by conventional solution phase methods using a fragment condensation strategy. The Boc group was used for N-terminal protection. Couplings were mediated by dicyclohexylcarbodiimide/1-hydroxybenzotriazole (DCC/HOBt). All of the intermediates were characterized by ${ }^{1} \mathrm{H}$ NMR spectroscopy, ${ }^{13} \mathrm{C}$ NMR spectroscopy and mass spectrometry. The final compounds were fully characterized by $500 \mathrm{MHz}, 400 \mathrm{MHz}{ }^{1} \mathrm{H}$ NMR spectroscopy, ${ }^{13} \mathrm{C}$ NMR spectroscopy (125 MHz, $100 \mathrm{MHz}$ ), mass spectrometry and IR spectroscopy.

\section{NMR Experiments}

All NMR experiments were performed on a Jeol $400 \mathrm{MHz}$ or Bruker $500 \mathrm{MHz}$ spectrometer. Compound concentrations were in the range of $1-10 \mathrm{mM}$ in $\mathrm{CDCl}_{3}$ and DMSO- $d_{6}$. 


\section{FT-IR Experiments}

FT-IR spectroscopy in the solid-state was performed with a PerkinElmer Spectrum RX1 spectrophotometer using the $\mathrm{KBr}$ disk method.

\section{Absorption spectroscopy}

The absorption spectra of peptides were recorded on a PerkinElmer UV/Vis spectrometer (Lambda 35) using a quartz cell having a $1 \mathrm{~cm}$ path length.

\section{Mass spectrometry}

Mass spectrometry was carried out on a Waters Corporation Q-Tof Micro YA263 high-resolution mass spectrometer by electrospray ionization (positive-mode).

\section{Polarised optical microscope}

A small amount of gel or solution of the compound was placed on a clean glass cover slip and then dried by slow evaporation, and then visualized at $40 \times$ magnification (an Olympus optical microscope equipped with a polarizer and CCD camera).

\section{Field emission scanning electron microscopy}

Field emission-scanning electron microscopy (FE-SEM) was performed to examine the morphologies of the synthesized peptides. A drop of peptide solution or gel was cast on a clean microscopic glass slide and dried under vacuum. The samples were gold-coated, and the images were captured in an FE-SEM apparatus (Jeol scanning microscope-JSM-6700F).

\section{Gelation}

$10 \mathrm{mg}$ of compound was dissolved in $1 \mathrm{~mL}$ of various organic solvents like different aromatic solvents like xylene, $o$-xylene, $p$-xylene, $m$-xylene, toluene, benzene (electron donating solvents) and chlorobenzene, 1,2-dichlorobenzene (electron withdrawing solvents) and different saturated hydrocarbons like petrol, diesel, kerosene, mustard oil, body oil, olive oil, and crude oil by the heating-cooling technique or sonication. Homogeneous gel was observed in hydrocarbons after sonication only.

\section{Rheology Experiments}

To examine the mechanical strength of the gel, we have performed rheological measurements on a MCR 102 rheometer (Anton Paar, modular compact rheometer) with a steel parallel plate geometry having $40 \mathrm{~mm}$ diameter at $20{ }^{\circ} \mathrm{C}$. The rheometer was attached to a Peltier circulator thermo cube in order to control the temperature accurately. The storage modulus $\left(G^{\prime}\right)$ and loss modulus $\left(G^{\prime \prime}\right)$ of the gel were then recorded by using the setup.

\section{X-Ray crystallography}

Diffraction quality white crystals of peptide mimetic 2 were obtained from methanol-water solution by slow evaporation. Intensity data were collected using a Bruker APEX-2 CCD diffractometer with MoK $\alpha$ radiation. Data were processed using the Bruker SAINT package. The structure solution and refinement were performed by SHELX97. Refinement of non-hydrogen atoms was performed using anisotropic thermal parameters. Crystal data of peptide 2: $\mathrm{C}_{20} \mathrm{H}_{29} \mathrm{~N}_{3} \mathrm{O}_{2}, M_{\mathrm{W}}=343.4$, $\mathrm{P} 1, a=9.5411(5) \AA, \quad b=9.5780(5) \AA, c=11.3793(6) \AA$, $\alpha=112.82^{\circ}, \beta=96.13^{\circ}, \gamma=99.65^{\circ}, V=927.87(10) \AA^{3}, Z=18$, $d_{\mathrm{m}}=1.229 \mathrm{Mg} \mathrm{m}^{-3}, R_{1}=0.1152$ and $\mathrm{w} R_{2}=0.2709$. CCDC $\dagger 2006754$ contains the crystallographic data for the peptide mimetic 2 .

\section{Conclusions}

In conclusion, we have reported the effect of isomerism on the structure, self-assembly and properties of two peptide mimetics. From X-ray crystallography, it was observed that the $p$-isomer adopted a kink-like conformation stabilized by $\mathrm{C}-\mathrm{H} \cdots \pi$ interactions and formed a supramolecular anti-parallel duplex and a layer by layer sheet-like structure in higher order assembly. However, only the $m$-isomer formed sonication responsive gels in different hydrocarbons such as hexane-EtOAc (19:1), diesel, kerosene, body oil and coconut oil. From rheology experiments, the gel was found to have physical cross-links and to be elastic in nature. The FE-SEM images depicted that the $m$-isomer exhibited nanofiber network morphology, whereas the $p$-isomer exhibited polydisperse microsphere morphology. The gel was found to be highly sensitive to alcohol vapor, although the kinetics become much slower from methanol to ethanol to isopropanol. The gel is also very responsive to acid vapors like $\mathrm{HCl}$. So, the peptide mimetic gel can be used as a low cost, room temperature easy to use material for alcohol and volatile acid vapor sensing. The results are promising and will be helpful for tuning and constructing functional supramolecular materials.

\section{Conflicts of interest}

There are no conflicts to declare.

\section{Acknowledgements}

We acknowledge IISER Kolkata, India, for analytical facilities. D. Chattapadhyay thanks INSPIRE, DST, India, for fellowship. S. K. Nandi thanks CSIR, India, for research fellowship.

\section{Notes and references}

1 E. V. Schneidemesser, P. S. Monks, J. D. Allan, L. Bruhwiler, P. Förster, D. Fowler, A. Lauer, W. T. Morgan, P. Paasonen, M. Righi, K. Sindelarova and M. A. Sutton, Chem. Rev., 2015, 115, 3856-3897.

2 W. J. Liu, W. W. Li, H. Jiang and H. Q. Yu, Chem. Rev., 2017, 117, 6367-6398.

3 D. M. Pace and A. Elliott, Cancer Res., 1962, 22, 107-112.

4 D. E. Moody, Drug Chem. Toxicol., 1991, 14, 319-342.

5 J. Reyes and R. Latorre, Biophys. J., 1979, 28, 259-280.

6 M. T. Smith, Annu. Rev. Public Health, 2010, 31, 133-148.

7 M. Hu, W. Kang, B. Cheng, Z. Li, Y. Zhao and L. Li, Chem. Eng. J., 2004, 102, 99-104.

8 Z. Xia, Biomimetic Principles and Design of Advanced Engineering Materials, John Wiley \& Sons, Ltd, Chichester, UK, 2016, pp. 188-209. 
9 (a) L. Fabbrizzi, M. Licchelli and P. Pallavicini, Acc. Chem. Res., 1999, 32, 846-853; (b) W. Shi, S. He, M. Wei, D. G. Evans and X. Duan, Adv. Funct. Mater., 2010, 20, 3856-3863; (c) G. De Santis, L. Fabbrizzi, M. Licchelli, N. Sardone and A. H. Velders, Chem. - Eur. J., 1996, 2, 1243-1250; (d) M. Irie, T. Fukaminato, T. Sasaki, N. Tamai and T. Kawai, Nature, 2002, 420, 759-760; (e) V. Fasano, M. Baroncuni, M. Moffa, D. Iandolo, A. Camposeo, A. Credi and D. Pisignano, J. Am. Chem. Soc., 2014, 136, 14245-14254; $(f)$ S. Uchiyama, N. Kawai, A. P. de Silva and K. Iwai, J. Am. Chem. Soc., 2004, 126, 3032-3033; (g) P. Duan, X. Zhu and M. Liu, Chem. Commun., 2011, 47, 5569-5571; (h) Z. Liu, Y. Jiang, J. Jiang, D. Zhai, D. Wang and M. Liu, Soft Matter, 2020, 16, 4115-4120.

10 (a) X. Zhang, H. Gorohmaru, M. Kadowaki, T. Kobayashi, T. Ishi-i, T. Thiemann and S. Mataka, J. Mater. Chem., 2004, 14, 1901-1904; (b) Q. Liu, J. Peng, L. Sun and F. Li, ACS Nano, 2011, 5, 8040-8048.

11 S. Srinivasan, P. A. Babu, S. Mahesh and A. Ajayaghosh, J. Am. Chem. Soc., 2009, 131, 15122-15123.

12 A. P. de Silva, H. Q. N. Gunaratne, T. Gunnlaugsson, A. J. M. Huxley, C. P. McCoy, J. T. Rademacher and T. E. Rice, Chem. Rev., 1997, 97, 1515-1566.

13 F. Bertani, N. Riboni, F. Bianchi, G. Brancatelli, E. S. Sterner, R. Pinalli, S. Geremia, T. M. Swager and E. Dalcanale, Chem. Eur. J., 2016, 22, 3312-3319.
14 F. Maffei, P. Betti, D. Genovese, M. Montalti, L. Prodi, R. D. Zorzi, S. Geremia and E. Dalcanale, Angew. Chem., Int. Ed., 2011, 50, 4654-4657.

15 F.-Y. Yi, Y. Wang, J.-P. Li, D. Wu, Y.-Q. Lan and Z.-M. Sun, Mater. Horiz., 2015, 2, 245-251.

16 S. Pramanik, C. Zheng, X. Zhang, T. J. Emge and J. Li, J. Am. Chem. Soc., 2011, 133, 4153-4155.

17 Z. Fei, N. Kocher, C. J. Mohrschladt, H. Ihmels and D. Stalke, Angew. Chem., Int. Ed., 2003, 42, 783-787.

18 K. Naka, T. Kato, S. Watase and K. Matsukawa, Inorg. Chem., 2012, 51, 4420-4422.

19 E. Takahashi, H. Takaya and T. Naota, Chem. - Eur. J., 2010, 16, 4793-4802.

20 Y. Matsunaga and J.-S. Yang, Angew. Chem., Int. Ed., 2015, 54, 7985-7989.

21 T. Das, A. Pramanik and D. Haldar, Sci. Rep., 2017, 7, 40485. 22 S. K. Nandi, K. Maji and D. Haldar, ACS Omega, 2018, 3, 3744-3751.

23 S. K. Nandi, S. R. Chowdhury, D. Podder, P. Ghorai and D. Haldar, Cryst. Growth Des., 2020, 20, 1884-1890.

24 S. K. Maity, S. Maity, P. Jana and D. Haldar, Chem. Commun., 2012, 48, 711-713.

25 S. K. Maity, S. Bera, A. Paikar, A. Pramanik and D. Haldar, Chem. Commun., 2013, 49, 9051-9053.

26 D. Podder, S. R. Chowdhury, S. K. Nandi and D. Haldar, New J. Chem., 2019, 43, 3743-3749. 